\title{
Research on the Sound Insulation Properties of Membrane-type Acoustic Metamaterials
}

\author{
Jinyu Hao ${ }^{1}$, Sheng Guo², Jian Cheng², Zhaopin $\mathrm{Hu}^{3}$, Hongyu Cui ${ }^{3 *}$ \\ ${ }^{1}$ Military Representative Office of Dalian 426 factory, Dalia, Liaoning, 116005, China \\ ${ }^{2}$ China Ship Development and Design Center, Wuhan, Hubei, 430064, China \\ ${ }^{3}$ School of Naval Architecture and Ocean Engineering, Dalian University of Technology, Dalian, Liaoning, 116024, China
}

\begin{abstract}
Low- and medium-frequency noise from ship cabins is difficult to control effectively. Excessive noise can seriously affect the acoustic stealth performance of ships. A novel membrane-type acoustic metamaterial is proposed in this paper with light weight and good sound insulation performance at low frequencies. The sound insulation performance of the metamaterial structure is analysed by using the acousticsolid coupling module in COMSOL software. Then, the ability to change the sound insulation performance of membrane-type acoustic metamaterials with cell structure and material parameters is obtained. The research results in this paper provide powerful technical support for noise control in ship cabins.
\end{abstract}

\section{Introduction}

Radiating noise from ships not only affects the working distance and accuracy of ship detection systems but also reduces their acoustic stealth performance. Due to the slow attenuation of low-frequency noise and its propagation distance in water, the effective control of lowfrequency noise is essential [1].

Traditional sound insulation materials, which follow the law of mass action, are not effective unless the material has a certain thickness. The effective isolation of low- and medium-frequency noise is often achieved at the cost of increasing the weight of the material, which is difficult to achieve when designing noise protection for ships, which require lightweight structures and compact spaces. Because acoustic metamaterials must confront the limitation of the law of mass action, membrane-type acoustic metamaterials have displayed advantages of light weight, small volume and flexible arrangement and have exhibited good sound insulation performance in the lowfrequency range, making them a research topic of interest [2]. In 2008, Yang et al. proposed a thin film acoustic metamaterial with an acoustic bandgap in a frequency range of 200-300 Hz [3]. Subsequently, other researchers studied the structure of membrane-type acoustic metamaterials, designed acoustic metamaterials with different structures, and explored their acoustic isolation properties [4-6]. It can be seen that membrane-type acoustic metamaterials exhibit excellent performance in the field of low-frequency sound insulation and vibration damping and provide a new path for low-frequency vibration and noise reduction protection in naval structures.

In this paper, a membrane-type metamaterial structure is designed, and the variation patterns of sound insulation under different structural and material parameters are discussed.

\section{Membrane-type acoustic metamaterial structure and calculation method}

\subsection{Cell structure}

To achieve effective low-frequency sound insulation properties, membrane-type acoustic metamaterial cell structures are generally composed of a membrane, a mass and a solid framework, shown in Figure 1 as a square metamaterial structure, where the side length is $l$, the membrane thickness is $t_{1}$ and the additional mass is a circular cross-section with a diameter of $d$. The height is $h$. When sound waves pass through this structure, due to resonance and anti-resonance between the sound waves and the membrane at certain frequencies, a certain sound insulation effect is produced. 


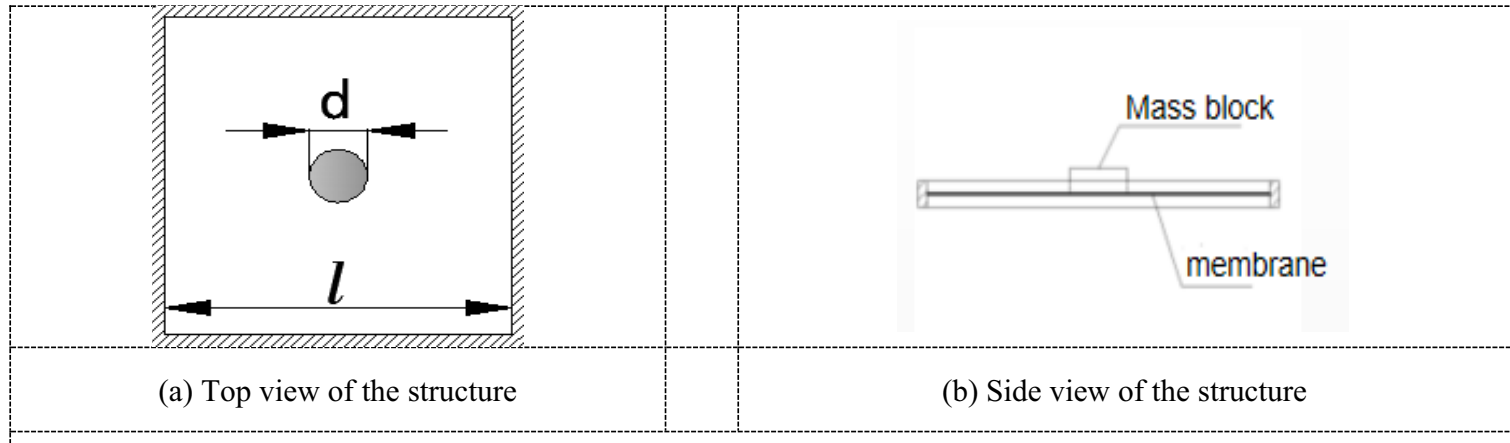

Figure 1. Cell structure model

\subsection{Calculation method}

Based on the structural dimensions and parametric properties of the membrane-type acoustic metamaterial, the modal superposition method was applied to calculate the sound insulation properties of the structural unit[7]. The equations of motion for the membrane structure with mass blocks are

In the formula,

$$
\rho_{S} \frac{\partial^{2} \omega}{\partial^{2} \mathrm{t}}+\rho_{\text {mass }} \mathrm{h}\left(x, y, x_{0}, y_{0}, l_{x}, l_{y}\right) \frac{\partial^{2} \omega}{\partial t^{2}}-T \nabla^{2} \omega=p_{i}+p_{r}+p_{t}
$$

$$
\hbar\left(x, y, x_{0}, y_{0}, l_{x}, l_{y}\right)=\left[\mathrm{H}\left(x-x_{0}\right)-\mathrm{H}\left(x-x_{0}-r\right)\right]\left[\mathrm{H}\left(y-y_{0}\right)-\mathrm{H}\left(y-y_{0}-r\right)\right]
$$

$$
\nabla^{2}=\frac{\partial^{2}}{\partial x^{2}}+\frac{\partial^{2}}{\partial y^{2}}
$$

where $\rho_{s}$ is the surface density of the membrane, $\rho_{\text {mass }}$ is the surface density of the mass block, $T$ is the membrane tension, $p_{i}$ is the incident sound pressure, $p_{r}$ is the reflected sound pressure, $p_{t}$ is the transmitted sound pressure, $H$ is the Heaviside function and $\omega(x, y, t)$ is the transverse displacement of a point on the membrane.

Using modal superposition theory, $\omega(x, y, t)$ can be written as a modal function $W_{n}(x, y)$ multiplied by the corresponding time function $q_{n}(t)$.

$$
\omega(x, y, t)=\sum_{n=1}^{N} W_{n}(x, y) q_{n}(t)
$$

The membrane is rigidly fixed around a solid frame, and then the modal equation can be written as

$$
W_{n}(x, y)=\sin \frac{r \pi}{L_{x}} x \sin \frac{r \pi}{L_{y}} y
$$

where

$$
r=1,2, \ldots, \mathrm{N}_{x} ; \quad \mathrm{s}=1,2, \ldots, \mathrm{N}_{y} ; n=\mathrm{N}_{y}(r-1)+s
$$

The membrane exhibits simple harmonic motion under sonic excitation, and $q_{n}(t)$ is written in plural form as

$$
q_{n}(t)=\tilde{q}_{n} e^{j \omega t}
$$

Substituting equations (4) and (6) into equation (1), the equation for the vibration of a membrane under acoustic excitation is expressed as

$$
-\omega^{2}\{[M]+[Q]\}\{\tilde{q}\}+j \omega[Q]\{\tilde{q}\}+[K]\{\tilde{q}\}=2 \tilde{p}_{z} \mathrm{H}
$$

When $L_{x}=L_{y}=l_{\text {is substituted into equation (7), }}$ the transmission coefficient $t_{p}$ is expressed as

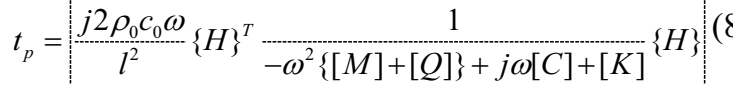

Then, the expression of the sound transmission loss TL of the cell is calculated as

$$
T L=20 \log _{10}\left(1 / t_{p}\right)
$$

\section{Sound insulation characteristics analysis of the membrane-type acoustic metamaterial structure}

\subsection{Sound insulation calculation of membrane- type metamaterial structures}

In this paper, COMSOL Multiphysics 5.4 large multiphysics field finite element software is used to numerically simulate the sound insulation of the metamaterial cellular structure. The membrane is made of silicone rubber, the edge length is $l=30 \mathrm{~mm}$, the thickness is $\mathrm{t}_{1}=0.2 \mathrm{~mm}$, and the applied prestress is $\mathrm{T}=200 \mathrm{~N} / \mathrm{m}$; the mass is made of an $\mathrm{NdFeB}$ magnet, the diameter is $5 \mathrm{~mm}$, the height is $2 \mathrm{~mm}$, and the mass is $0.15 \mathrm{~g}$; the frame is made of aluminium, the corresponding material parameters of each material are shown in Table 1, and the 
finite element model is composed of a membrane acoustic metamaterial unit and an air domain on both sides. The solid frame structure is surrounded by a fixed constraint, and the boundary conditions between the membrane and the solid frame and the mass block are set as displacement constraints. The outermost part of the air domain is set as a perfectly matched layer to eliminate the effect of acoustic reflection on the results. The remaining peripheral boundary conditions are uniformly set as hard acoustic field boundary conditions. The calculated sound isolation curves for the metamaterial structure in the range of $100-1000 \mathrm{~Hz}$ are shown in Figure 2.

Table 1. Characteristic table for each material

\begin{tabular}{ccccc}
\hline Names & Materials & $\begin{array}{c}\text { Density } \\
\left(\mathrm{kg} / \mathrm{m}^{3}\right)\end{array}$ & $\begin{array}{c}\text { Modulus of elasticity } \\
(\mathrm{Pa})\end{array}$ & Poisson's ratio \\
\hline Membrane & Silicone rubber & 980 & $2 \times 10^{5}$ & 0.49 \\
Mass block & NdFeB magnet & 7400 & $1.6 \times 10^{11}$ & 0.28 \\
Frame & Aluminium & 2730 & $6.9 \times 10^{10}$ & 0.33 \\
\hline
\end{tabular}

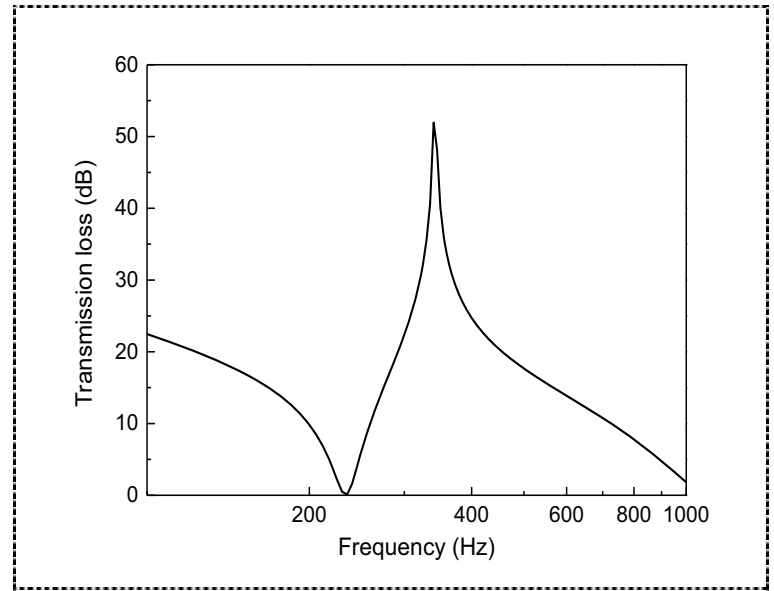

Figure 2. Sound insulation curve for metamaterials.

In Figure 2, it can be seen that the metamaterial structure has a sound isolation peak at $340 \mathrm{~Hz}$ with $52 \mathrm{~dB}$ isolation and an average isolation of $20 \mathrm{~dB}$ in the isolation bandgap of $300-700 \mathrm{~Hz}$.

\subsection{Factors influencing the amount of sound insulation for membrane-type acoustic metamaterial structures}

The changes in the structural parameters of membranetype acoustic metamaterials have a great influence on their sound insulation properties, and the structural sound insulation curves obtained by varying the membrane prestress, membrane thickness, membrane edge length and mass block distribution positions were calculated and analysed, as shown in Figure 3(a)-(d).

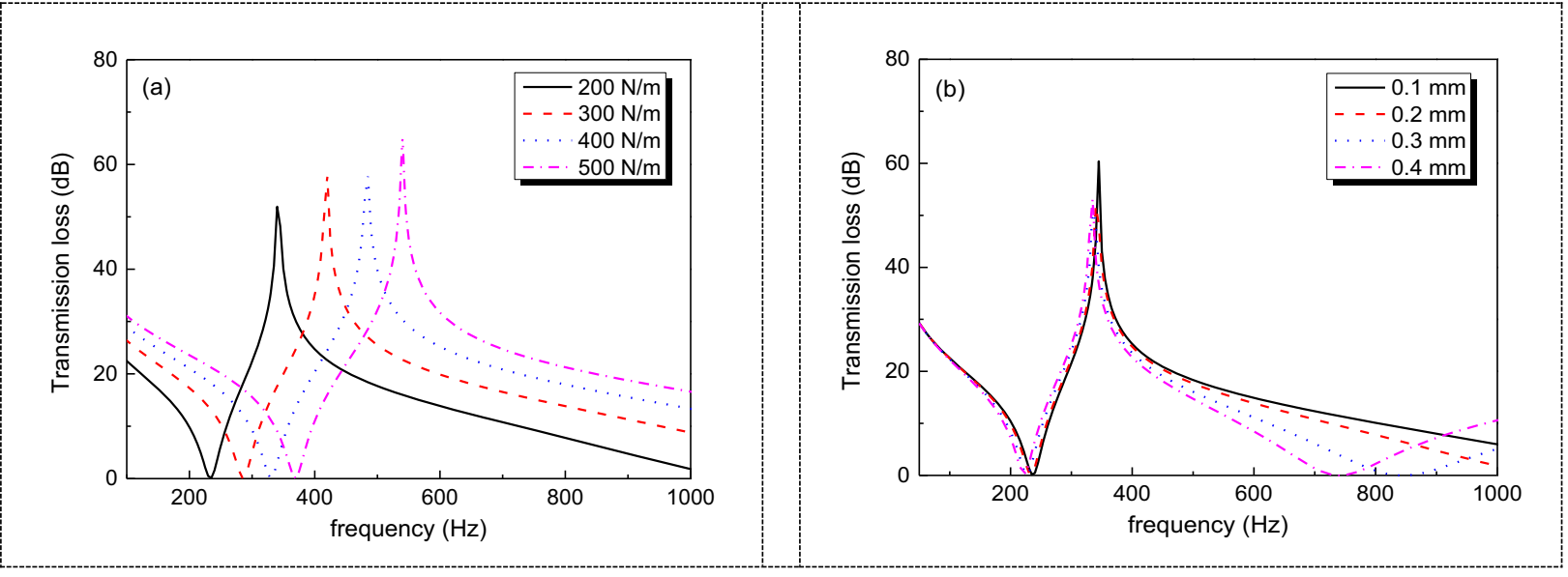




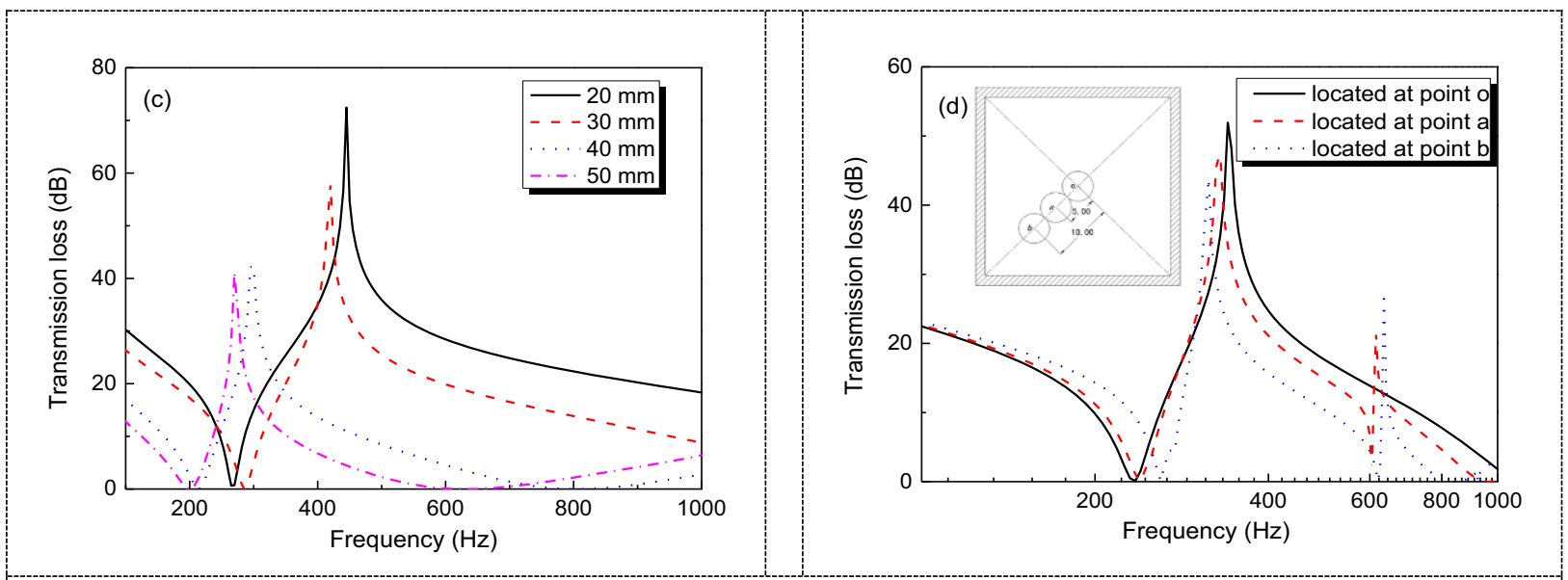

Figure 3. Relationship between sound insulation and material and structural parameters

Figure 3(a) shows the acoustic metamaterial sound isolation performance curve for different cases of membrane prestress. It can be seen in the figure that with the gradual increase in the membrane prestress, the entire sound insulation volume curve shifts towards high frequencies, and the sound insulation volume gradually increases.

Figure 3(b) shows the acoustic metamaterial sound isolation performance curve for different membrane thicknesses. From the data in the figure, it can be seen that as the thickness of the membrane gradually increases, the frequencies corresponding to the acoustic isolation peaks of the material move towards high frequencies, the frequencies corresponding to the first acoustic isolation peak and the first acoustic isolation valley are basically unchanged, and the second acoustic isolation peak and the second acoustic isolation valley as a whole move towards low frequencies.

Figure 3(c) shows the acoustic metamaterial sound insulation performance curve for different edge length dimensions of the membrane. From the data in the figure, it can be seen that as the size of the membrane increases, the amount of sound insulation of the material gradually decreases, and the first acoustic isolation peak and the first acoustic isolation valley both move towards lower frequencies. The smaller the size is, the larger the

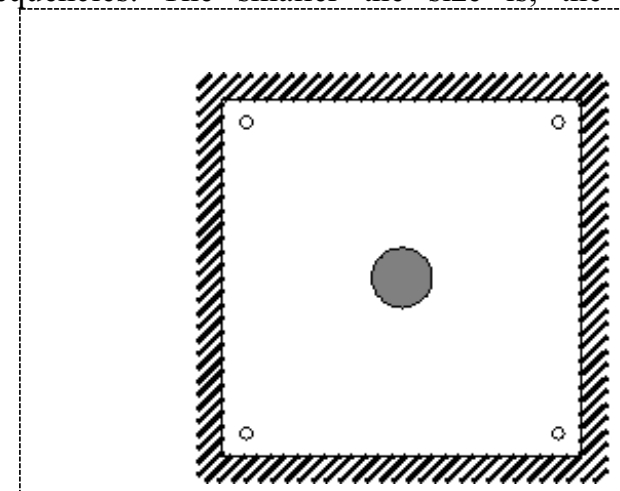

Figure 4. Basic structure of perforated membrane frequency band width and the higher the amount of sound insulation.

Figure 3(d) shows the acoustic isolation performance curves of the metamaterial structure at different placement positions of the mass block. In the process of moving the mass block from point $o$ to point $b$, the band width gradually decreases, the isolation peak gradually decreases, and the isolation peak moves towards lower frequencies. When the additional mass is located at the centre of the membrane, the maximum amount of sound insulation and the band width are optimal. As the mass moves from point o to point $\mathrm{a}$, the first diaphragm valley remains essentially unchanged, and the second diaphragm valley shifts towards lower frequencies.

\subsection{Research on the acoustic insulation properties of perforated membranes}

In this section, the acoustic isolation properties of the perforated membrane were studied, and simulations were carried out for the diameter, number and number of circles of the holes. The basic structure is shown in Figure 4. Figure 5 shows the acoustic metamaterial sound insulation performance curve when the diameter of the perforation is gradually increased from $0.02 \mathrm{~mm}$ to $0.08 \mathrm{~mm}$.

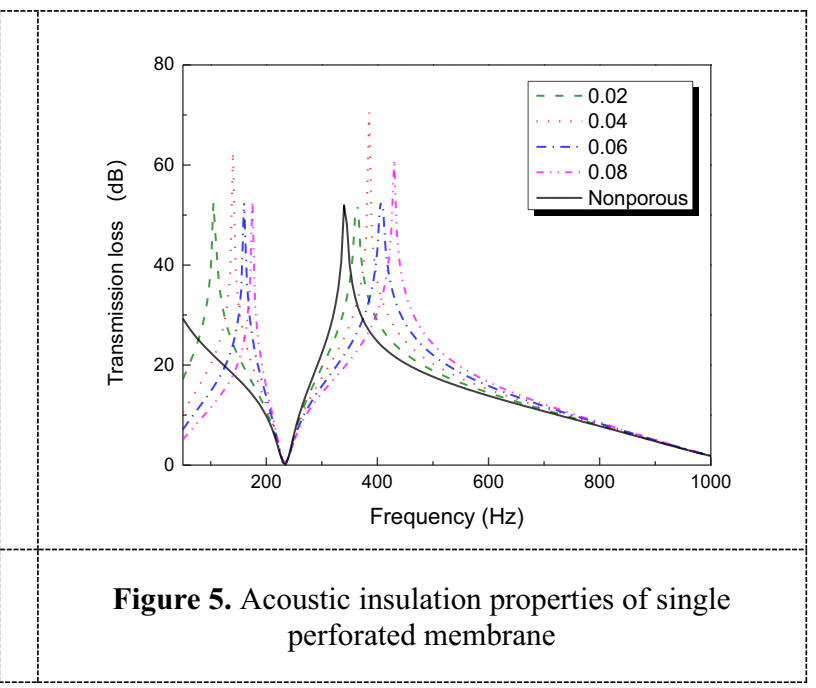


The solid line in Figure 5 shows the amount of sound isolation when there are no holes; there is a sole sound isolation peak at $340 \mathrm{~Hz}$. When the perforation is introduced, it can be seen that the metamaterial structure has an increased isolation peak below $200 \mathrm{~Hz}$, which indicates that the perforated membrane has better lowfrequency isolation performance than the nonperforated membrane. As the diameter of the perforation increases, the frequencies corresponding to the first and second sound-isolation peaks move towards high frequencies, and the frequencies corresponding to the first and second sound-isolation valleys are basically unchanged.

Based on the structure of the membrane with a 0.04 $\mathrm{mm}$ perforation diameter, the effect of diagonal placement of different numbers of holes on the sound insulation performance of the metamaterial is investigated, as shown in Figure 6 for the basic structure of the membrane metamaterial with different numbers of diagonal perforations. Figure 7 shows the acoustic insulation performance curves of the diagonal cloth with different numbers of holes placed in the membrane. It can be seen in Figure 7 that with the increase in diagonal perforations, the two peaks of the structure move towards the high frequencies, and the frequencies corresponding to the first and second valleys of sound insulation are basically unchanged. The sound insulation peak is largest when there is one hole in each corner of the square membrane structure.

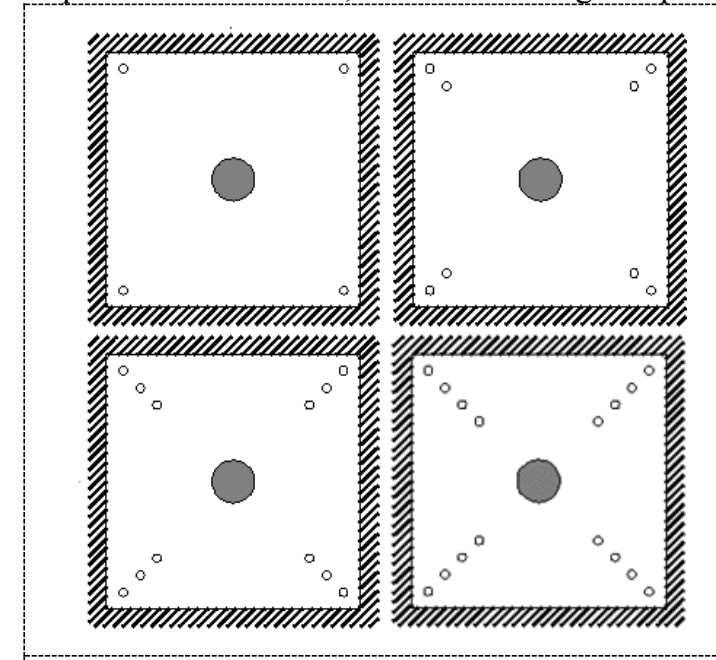

Figure 6. Basic structure with different number of diagonal perforations in membranes

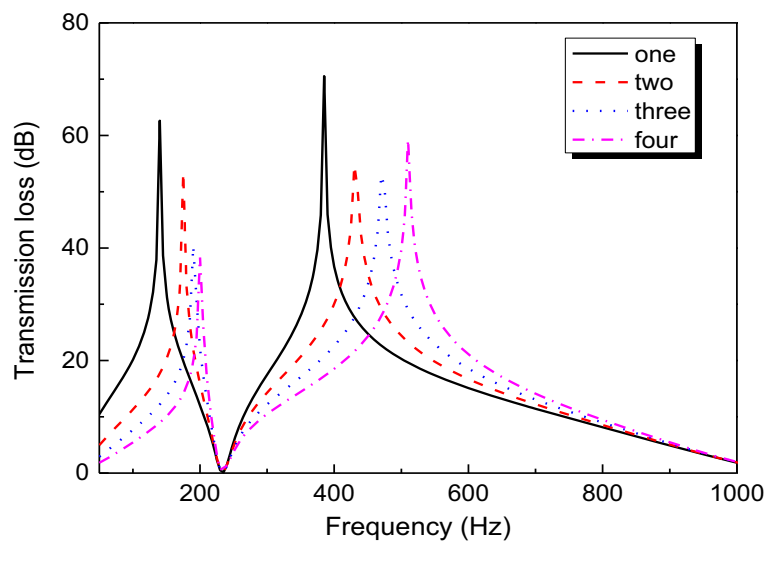

Figure 7. Diagonal number of holes for different membranes acoustic performance.
Based on the structure of the membrane with a 0.04 $\mathrm{mm}$ perforation diameter, the effect of placing different annular turns of holes on the sound insulation performance of the metamaterial is investigated, as shown in Figure 8.
Figure 9 shows the sound insulation performance curve of the membrane with different numbers of annular holes.

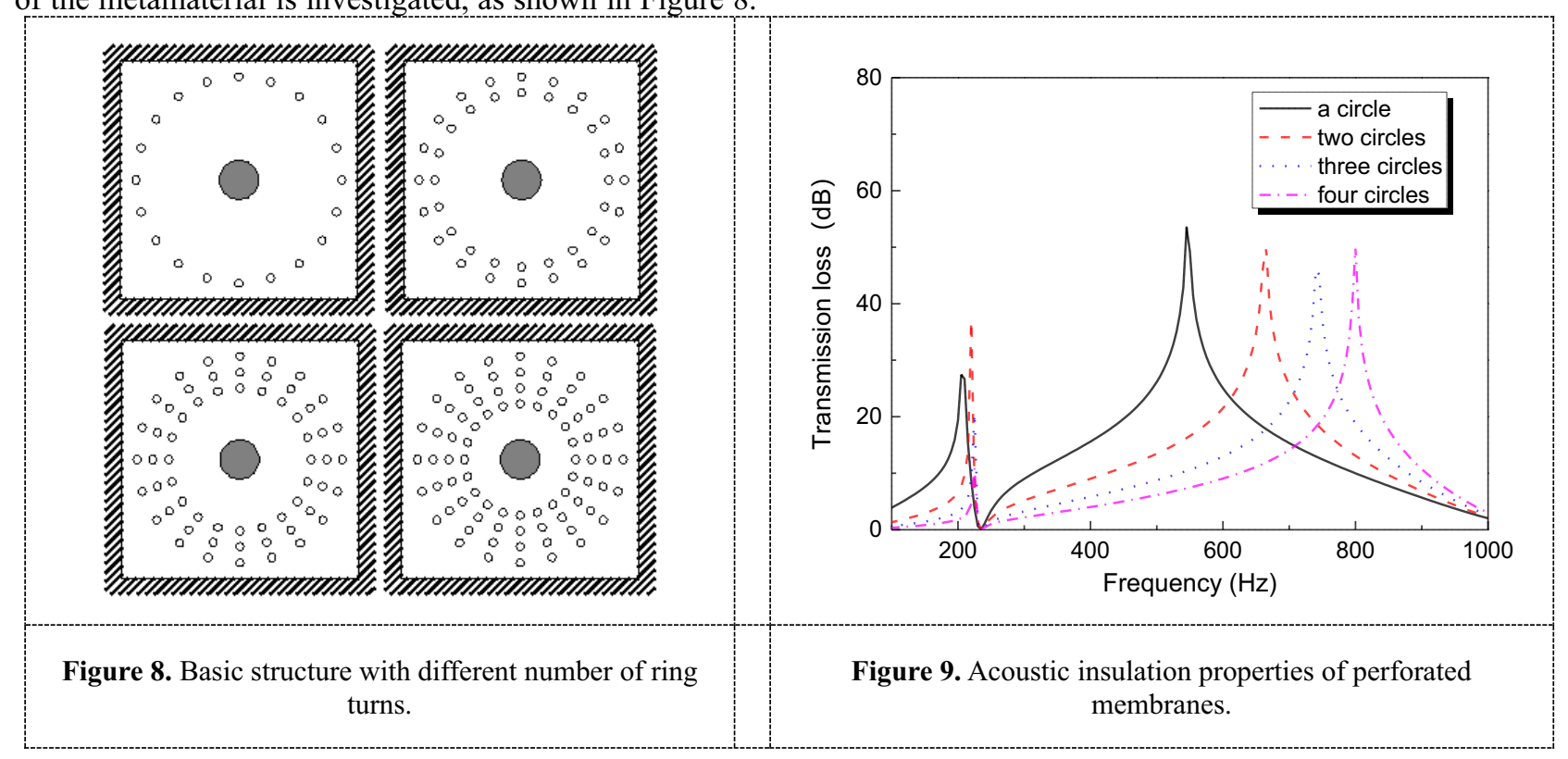

As seen in Figure 9, as the number of loops increases, the peaks of the two acoustic isolation peaks move towards high frequencies, the band width decreases, the peak value of the first acoustic isolation peak of the 
membrane metamaterial structure with two loops placed is the largest, and the frequencies corresponding to the first and second acoustic isolation valleys are basically unchanged.

\section{Conclusion}

In this paper, to meet the noise protection design requirements of ship cabins that need lightweight structures and compact spaces, based on the fact that membrane-type acoustic metamaterials have light weight and good low-frequency sound insulation performance, the acoustic-solid coupling module in COMSOL software was used to analyse the influence of the metamaterial structure and its insulation performance on parameters such as membrane prestress, membrane thickness, membrane edge length and mass distribution, and membrane acoustic metamaterials was obtained. The sound insulation properties of the acoustic metamaterials varied with the structural and material parameters of the cells. The perforated structure membrane metamaterial structure was designed, the diameter size, number of perforations and number of circumferential arrangement circles were investigated, and the sound insulation changes of the structure under different conditions of the designed structure were obtained. The research results of this paper provide powerful technical support for the noise control of ship compartments.

\section{References}

1. Li, Q., Yang, D. Q., Yu, Yang. (2018) Numerical calculation method of acoustic-solid coupling for low-frequency underwater radiation noise of ships. Vibration and Impact, 37(3):194-197.

2. Bolton, J.S., Shiau, N.M., Kang, Y.J. (1996) Sound transmission through multi-panel structures lined with elastic porous materials. Journal of Sound and Vibration, 191(3):317-347.

3. Yang, Z., Mei, J., Yang, M., et al. (2008) MembraneType Acoustic Metamaterial with Negative Dynamic Mass. Physical Review Letters, 101(20):204301.

4. Wu, Jiuhui., Ma, Fuyin., Zhang, Siwen., et al. (2016) A review of the application of acoustic metamaterials in low frequency vibration and noise reduction. Journal of Mechanical Engineering,52(13):68-78.

5. Lu, Zhenbo., Yu, Xiang., Lau, Siu-Kit., et al. (2020) Membrane-type acoustic metamaterial with eccentric masses for broadband sound isolation. Applied Acoustics, 157:107003.

6. Zhou, Guojian., Wu, Jiuhui., Lu, Kuan., et al. (2020) Broadband low-frequency membrane-type acoustic metamaterials with multi-state anti-resonances[J]. Applied Acoustics, 159:107078.

7. Zhang, Jialong., Yao, Hong., Du, Jun., et al. (2016) Analysis of structural sound insulation properties of membrane type acoustic metamaterial plates. Journal of Artificial Crystals, 45(10):2549-2555. 